\title{
A Case of Pulmonary Hyalinizing Granuloma
}

\author{
Yoshiki Anazawa, Hiromi Nagai, Masakichi Motomiya, \\ Toyoharu Isawa, Yasuki SaIto*, Tohru Takahashi $\dagger$ \\ and Yoshinori Kawabata
}

Departments of Internal Medicine, *Surgery, and $\dagger$ Pathology, the Research Institute for Tuberculosis and Cancer, Tohoku University, Sendai 980, and $\ddagger$ Department of Pathology, the Research Institute of Tuberculosis, Japan Anti-Tuberculosis Association, Kiyose 204

\begin{abstract}
Anazawa, Y., Nagai, H., Мотomiya, M., Isawa, T., Satto, Y., Takahashi, T. and Kawabata, Y. A Case of Pulmonary Hyalinizing Granuloma. Tohoku J. Exp. Med., 1992, 167 (1), 39-45 — A case of pulmonary hyalinizing granuloma was presented. The patient was a 37-year-old male who was found to have abnormal chest roentgenograms showing multiple pulmonary nodules taken at the annual chest mass survey in October 1989. The largest nodule measured $35 \mathrm{~mm}$ in diameter. He was asymptomatic. No definite diagnosis was established either by brushing cytology, biopsy through bronchoscopy or percutaneous needle biopsy. No abnormalities were found in the GI tract, the urogenital system or the bone. Brain CT indicated an iso-density area surrounded by low density in the left fronto-parietal region. Two nodules of the lingula removed by open lung biopsy revealed a homogeneous cut surface. Histological diagnosis was pulmonary hyalinizing granuloma, consisting of hyalinized collagen fibers and bundles infiltrated with chronic inflammatory cells. No therapeutic effect was recognized with prednisolone. Craniotomy was performed and the brain lesion was removed. Anaplastic astrocytoma was the diagnosis. The brain lesion had no etiological correlation with pulmonary hyalinizing granuloma. Pulmonary hyalinizing granuloma itself is a rare benign disease with no specific therapy and is important in the differential diagnosis of lung diseases showing multiple pulmonary nodules. benign pulmonary nodules; multiple pulmonary nodules; pulmonary hyalinizing granuloma
\end{abstract}

In 1977 Engleman et al. described pulmonary hyalinizing granuloma (PHG). Since then only one case has been reported in Japan (Kuramochi et al. 1985) and about 50 cases in other countries (Engleman et al. 1977 ; Drasin et al. 1979 ; Schlosnagle et al. 1982 ; Dent et al. 1983 ; Chalaoui et al. 1984 ; Guccion et al. 1984 ; Macedo and Adolph 1985 ; Maijub et al. 1985 ; Yousem and Hochholzer 1987; Gans et al. 1988). An immune response to the antigenic stimulus by infection or an auto-immune process has been postulated in the pathogenesis but the true etiology yet remains to be obscure. In this report we present a case report of a patient with $\mathrm{PHG}$ complicated with malignant brain tumor.

Received April 20, 1992; revision accepted for publication April 28, 1992. 


\section{Case Report}

A 37-year-old male was admitted to the hospital of The Research Institute for Tuberculosis and Cancer, Tohoku University on December 1, 1989 because of abnormal chest roentgenograms showing multiple nodular shadows. Either his past history or family history was non-contributory. He smoked 20 cigarettes a day for 20 years. He was

TABLE 1. Laborartory findings on admission

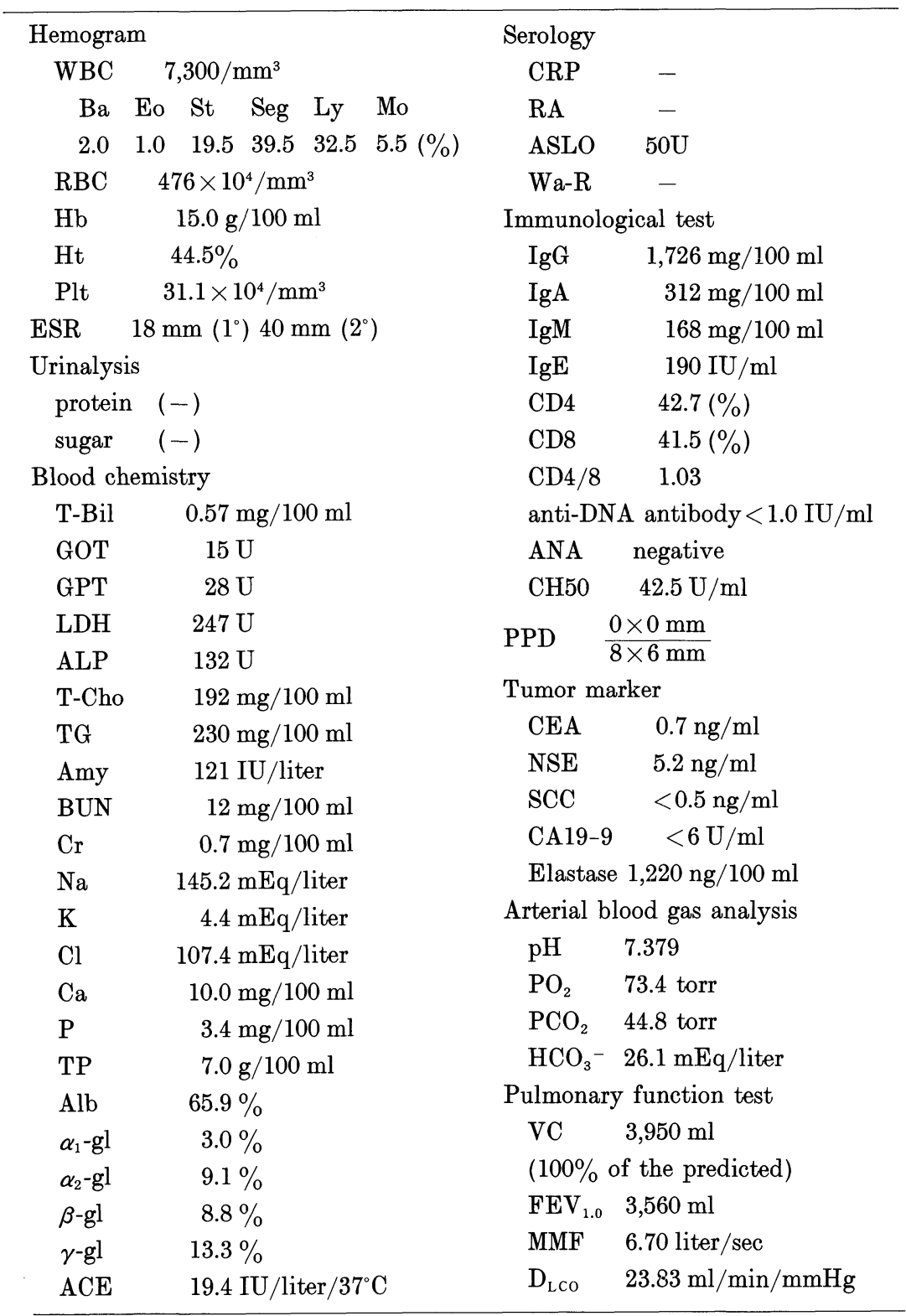


asymptomatic. The annual chest roentgenogram in 1987 was within normal limits. $\mathrm{He}$ did not take the annual examination of the chest in 1988.

He was $167.5 \mathrm{~cm}$ in height and $80 \mathrm{~kg}$ in weight. His temperature was $35.5^{\circ} \mathrm{C}$, pulse, $60 /$ min ; and blood pressure, $110 / 65 \mathrm{mmHg}$. Physical examination was essentially within normal limits; no conjunctival anemia nor jaundice, no palpable lymph nodes, no cyanosis of the lips or the nails, normal breath and heart sounds without arrhythmia, soft and flat abdomen without palpable liver, spleen or kidneys. Neither motor nor sensory disturbances were noted.

Laboratory findings are shown in Table 1 . White blood cells (WBC) were $7,300 / \mathrm{mm}^{3}$ with some shift to the left. Erythrocyte sedimentation rate (ESR) was $18 \mathrm{~mm} / \mathrm{hr}$. Arterial blood gases at room air showed that $\mathrm{pH}$ was $7.379, \mathrm{Po}_{2}, 73.4$ torr and $\mathrm{Pco}_{2}, 44.8$ torr. Pulmonary function test was normal. PPD skin reaction was $8 \times 6 \mathrm{~mm}$. Tumor markers were within normal range. Immunoglobulins, CD4 and CD8 were within normal range. Antinuclear antibody (ANA) and anti-DNA antibody were normal. CH50 was $42.5 \mathrm{U} / \mathrm{ml}$ and slightly elevated (normal $<40 \mathrm{U} / \mathrm{ml}$ ). Cryptococcal and echinococcal antibodies were negative. Microbiological examinations of sputum including acid fast bacilli were negative. Cytology of sputum revealed class II.

Plain chest roentgenograms on admission revealed multiple nodular shadows in both lungs (Fig. 1). The lesions were sharply circumscribed and homogeneous in density and the largest nodule measured $35 \mathrm{~mm}$ in diameter. CT scan of the thorax confirmed the x-ray findings (Fig. 2). No mediastinal lymphadenopathy was noted.

From the above findings metastatic lung cancer was most suspicious. Brushing cytology and biopsy through bronchoscopy and percutaneous needle biopsy were not diagnostic of malignancy. We investigated the GI tract, the urogenital and skeletal systems to search for a primary lesion if any, but no abnormalities were found. Brain computed tomography (CT) was also performed. Agaist our expectation brain CT indicated an iso-density area surrounded by low density in the left fronto-parietal region that was compatible with brain

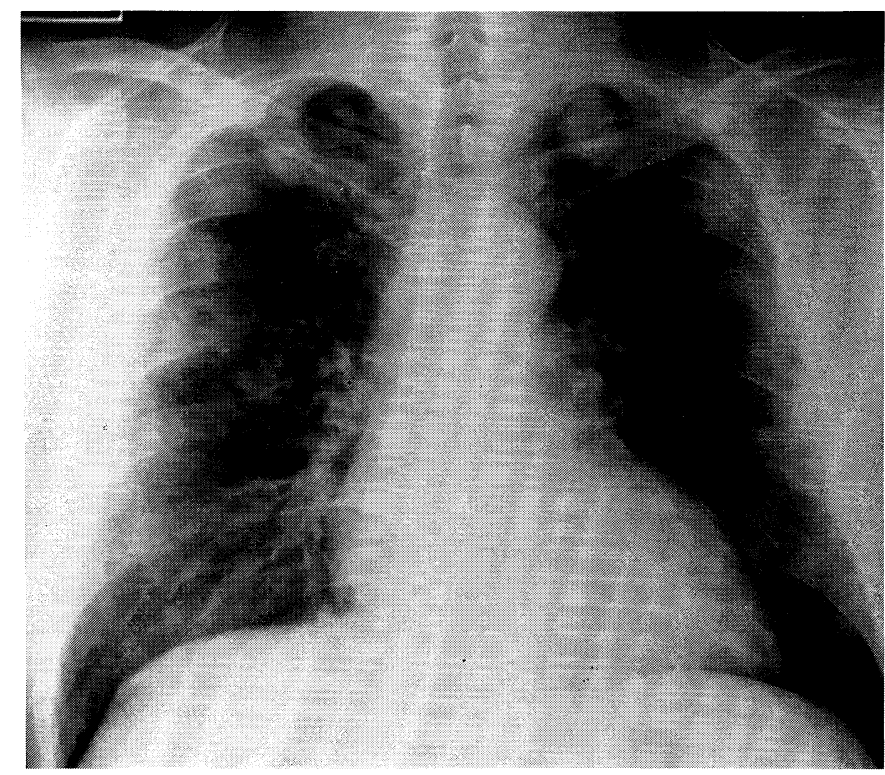

Fig. 1. Chest roentgenogram on admission (Dec. 1, 1989), showing multiple nodular densities in both lungs. 


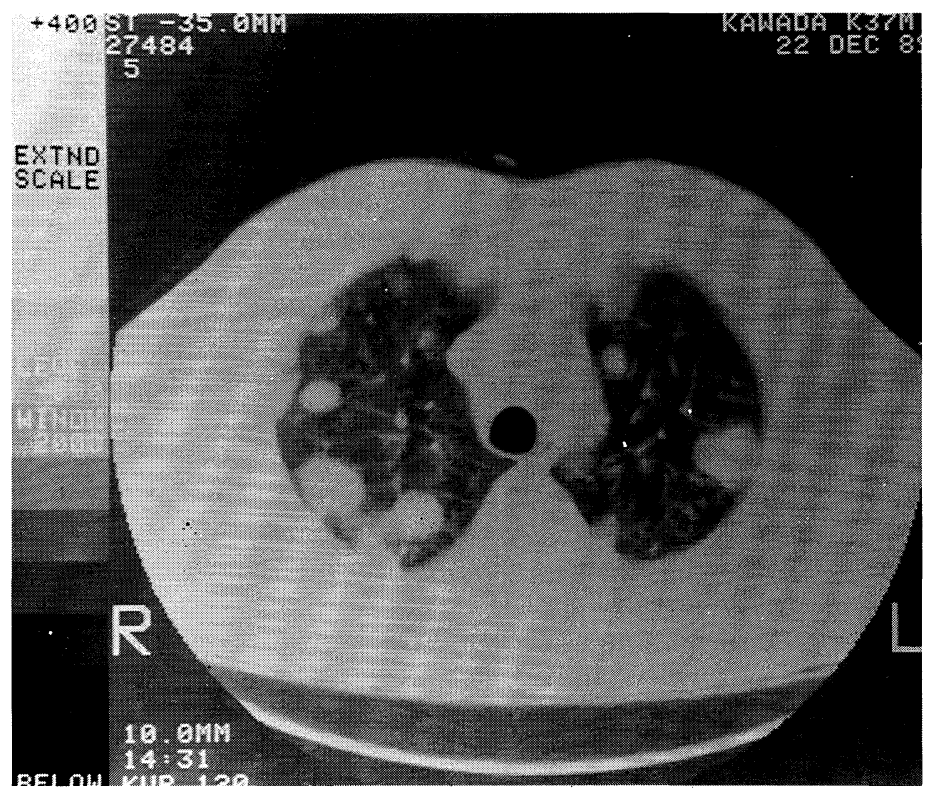

Fig. 2. CT scan of the thorax, showing multiple well circumscribed nodules.

metastasis. On February 27, 1990 he underwent an open lung biopsy and two nodules in the left lingula (S4) were removed. The nodules were well defined and cartilaginous hard. The cut surface was gray-white in color and homogeneous. Portions of the nodules were sent for microbiological, fungal and acid-fast stain and culture. All tests were negative.

Histopathological examinations revealed as follows; the margin of the nodule was sharply demarcated. Lamellar, thick collagen bundles or hyalinized fibrous materials with a few inflammatory cells could be seen in the central part of the lesion (Fig. 3). Chronic inflammatory cells consisting of lymphocytes were seen forming germinal centers, and the plasma cells and histiocytes infiltrated in the periphery of the nodule (Fig. 4). The histopathological diagnosis was compatible with pulmonary hyalinizing granuloma. It took almost one month and a half to reach the diagnosis.

He began to complain of a mild headache in March, 1990. He was given $40 \mathrm{mg}$ of oral prednisolone from April 20, 1990 over 4 months' period with tapering dose. There were neither changes on chest roentgenograms nor improvement in the mild headache. The brain lesion appeared slightly increased in size on subsequent brain CT scans. If it had something to do with the lung lesions, it should not grow in size in the time interval of several months. An exploratory craniotomy was performed on August 13, 1990 ; the brain lesion was removed. Histopathological diagnosis was anaplastic astrocytoma. After surgery 54 Gy of radiation to the whole brain and 4 courses of chemotherapy with nimustine (ACNU) were given in combination. At present (as of April 1992) he remains asymptomatic and no radiological changes on chest roentgenograms have been noticed.

\section{Discussion}

The chest roentgenograms of PHG frequently reveal multiple and bilateral pulmonary nodules. Radiographic differential diagnoses include metastatic lung cancer, rheumatoid nodules, plasma cell granuloma, Wegener's granulomatosis, 


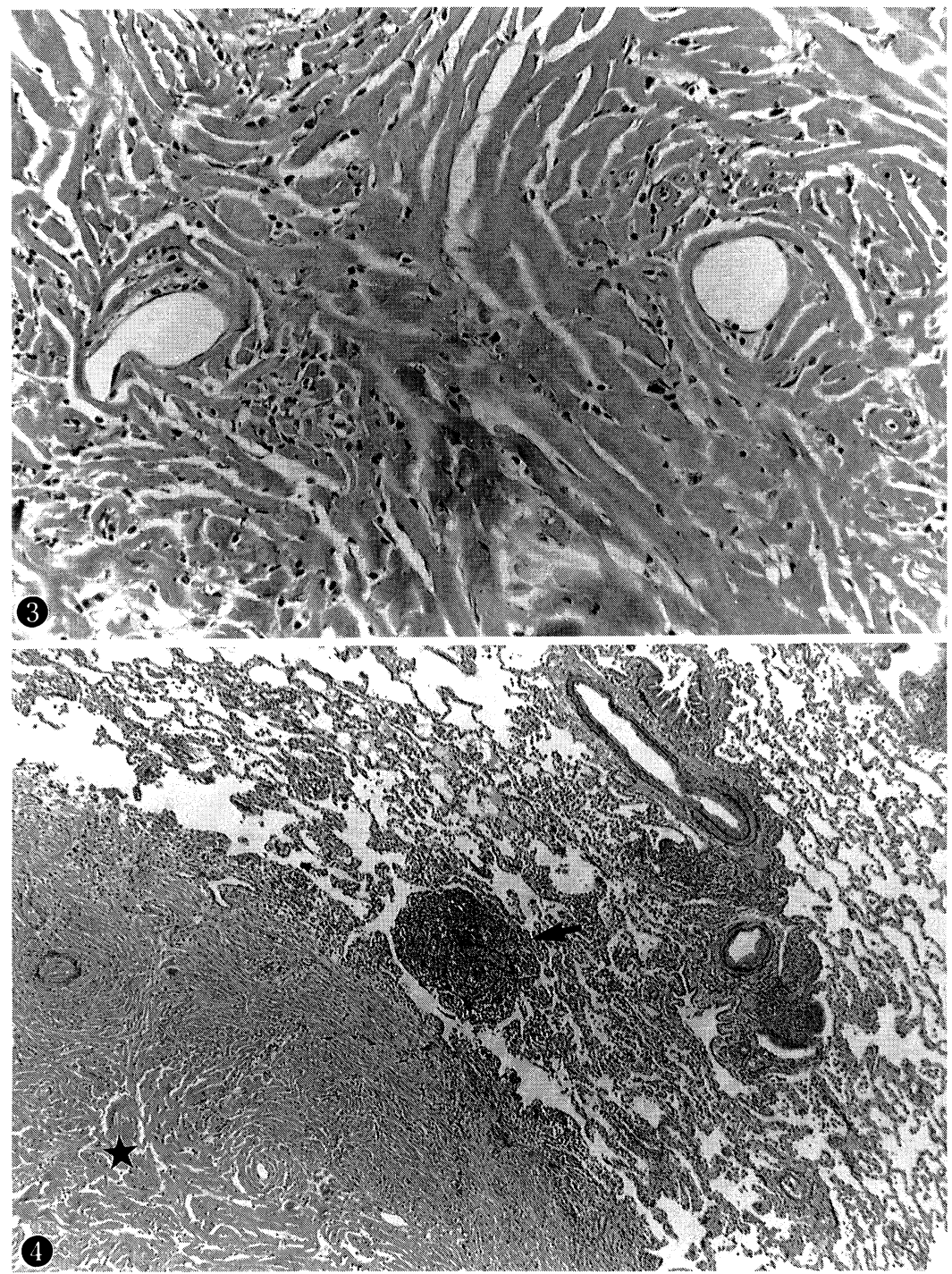

Fig. 3. Microphotograph of a pulmonary nodule, showing haphazard arrangement of hyalinized collagen fibers with chronic inflammatory cell infiltration. $(\mathrm{E}-\mathrm{M}$ stain, $\times 50$ )

Fig. 4. Microphotograph of an interface between pulmonary hyalinizing granuloma (asterisk) and pulmonary tissue. Note the dense aggregate of chronic inflammatory cells consisting mainly of lymphocytes forming a germinal center (arrow). (E-M stain, $\times 10$ ) 
multinodular sarcoidosis, nodular amyloidosis, histoplasmosis, echinococcosis and tuberculosis. All these lesions should be differentiated by their clinical, biological, bacteriological, radiological and histopathological characteristics (Kalifa et al. 1976 ; Engleman et al. 1977 ; Chalaoui et al. 1984).

Diagnosis of $\mathrm{PHG}$ can be made only by histopathological examination. PHG have unique histologic features. The lesions are well-circumscribed nodules. The center of the nodule is occupied by lamellae of hyalinized collagen. The deposition pattern of lamellar collagen is diagnostic of this disease and characterized by ropy whorled collagen bundles separated by clear spaces. The collagen is frequently arranged in parallel lamellae or in a coarse storiform pattern. Necrosis, calcification or ossification is rare. Electronmicroscopically the hyaline lamellae are consisted of an electron-dense, compact, homogeneous, amorphous material (Guccion et al. 1984). Blood vessels in the nodule are surrounded by the hyalinized collagen fibers and have intimal fibroelastosis and medial hyalinization. Toward the periphery of the nodule, the spaces between the lamellae are occupied by flattened, compressed fibroblasts and a sprinkling of mononuclear inflammatory cells, creating a distinct gradient of cellularity from the center to the periphery of the nodule. At the interface between the pulmonary parenchyma and the hyalinized nodule there are occasional aggregates of non-specific chronic inflammatory cells consisting mainly of lymphocytes forming germinal centers, and rarely of plasma cells and histiocytes (Maijub et al. 1985; Yousem and Hochholzer 1987).

PHG is usually radiologically characterized by multiple and bilateral nodules without preferential localization. Commonly the lesions are well defined and homogeneous. Calcifications or cavity formation is rare. Diameter of the nodules is variable from a few millimeters to $15 \mathrm{~cm}$. Patients with PHG are asymptomatic or present with mild symptoms of cough, fever, dyspnea and fatigue. The mean age of $\mathrm{PHG}$ is 45 years. There is no sex or race predilection (Engleman et al. 1977).

The etiology of $\mathrm{PHG}$ is unknown. The nodules probably represent an immune response to the antigenic stimulus by infection or an auto-immune process. Sometimes sclerosing mediastinitis or retroperitonitis is complicated with PHG (Engleman et al. 1977 ; Dent et al. 1983). The former has a microscopic picture much like that of PHG, and can be caused by granulomatous diseases like histoplasmosis or tuberculosis (Chalaoui et al. 1984). Previous exposures to fungal or mycobacterial diseases have been reported in some of patients with PHG (Engleman et al. 1977; Yousem and Hochholzer 1987). Guccion et al. (1984) and Schlosnagle et al. (1982) have reported immunologic abnormalities, including elevated ANA, rheumatoid factor and circulating immune complexes in their patients. Yousem and Hochholzer (1987) reported auto-immune phenomena indicated by positive ANA, positive rheumatoid factor, or Coombs-positive hemolytic anemia. Drasin et al. (1979) reported a case of 
PHG complicated with malignant lymphoma who developed multiple myeloma and systemic amyloidosis nine years later. In our case only serum complement titer was slightly elevated. No other immunologic abnormalities could be detected and no previous infection seemed responsible for the present illness. Chest roentgenograms two years previous to the present episode were within normal limits.

The prognosis of PHG is generally favorable. Normal longevity is common. Usually the nodules tend to grow slowly, but spontaneous regression and a status quo are also described (Macedo and Adolph 1985). No specific therapy for PHG has been described. Schlosnagle et al. (1982) reported a case that nodules did not change after treating with prednisolone. We also administered prednisolone to our patient in vain. The brain lesion in the present case did not seem to have any etiological correlation with PHG.

PHG should be remembered in the differential diagnosis of lung diseases showing multiple pulmonary nodules.

\section{References}

1) Chalaoui, J., Grégoire, P., Sylvestre, J., Lefebvre, R. \& Amyot, R. (1984) Pulmonary hyalinizing granuloma: A cause of pulmonary nodules. Radiology, 152, 23-26.

2) Dent, R.G., Godden, D.J., Stovin, P.G.I. \& Stark, J.E. (1983) Pulmonary hyalinizing granuloma in association with retroperitoneal fibrosis. Thorax, 38, 955-956.

3) Drasin, H., Blume, M.R., Rosenbaum, E.H. \& Klein, H.Z. (1979) Pulmonary hyalinizing granulomas in a patient with malignant lymphoma, with development nine years later of multiple myeloma and systemic amyloidosis. Cancer, 44, 215-220.

4) Engleman, P., Liebow, A.A., Gmelich, J. \& Friedman, P.J. (1977) Pulmonary hyalinizing granuloma. Am. Rev. Respir. Dis., 115, 997-1008.

5) Gans, S.J.M., van der Elst, A.M.C. \& Straks, W. (1988) Pulmonary hyalinizing granuloma. Eur. Respir. J., 1, 389-391.

6) Guccion, J.G., Rohatgi, P.K. \& Saini, N. (1984) Pulmonary hyalinizing granuloma. Electron microscopic and immunologic studies. Chest, 85, 571-573.

7) Kalifa, L.G., Schimmel, D.H. \& Gamsu, G. (1976) Multiple chronic benign pulmonary nodules. Radiology, 121, 275-279.

8) Kuramochi, S., Kawai, K., Yakumaru, K., Mikata, S., Torigata, O., Kasuga, Y. \& Fujiwara, T. (1985) An autopsy case with systemic fibrosis of unknown origin complicating pulmonary hyalinizing granuloma (Engleman, P. et al. 1977). Acta Pathol. Jpn., 74, 300-301.

9) Macedo, E.V. \& Adolph, J. (1985) Pulmonary hyalinizing granulomas. J. Can. Assoc. Radiol., 36, 66-67.

10) Maijub, A.G., Giltman, L.I., Verner, J.L. \& Peace, R.J. (1985) Pulmonary hyalinizing granuloma. Ann. Allergy, 54, 227-229.

11) Schlosnagle, D.C., Check, I.J., Sewell, C.W., Plummer, A., York, R.M. \& Hunter, R.L. (1982) Immunologic abnormalities in two patients with pulmonary hyalinizing granuloma. Am. J. Clin. Pathol., 78, 231-235.

12) Yousem, S.A. \& Hochholzer, L. (1987) Pulmonary hyalinizing granuloma. Am. J. Clin. Pathol., 87, 1-6. 